\title{
A Pilot Study of Client Outcomes from Exercise Physiology in a Youth Mental Health Service
}

\begin{abstract}
Aim

The aim of this pilot study was to evaluate the utilisation and experience of an exercise physiology program, known as Bod Squad at a youth mental health service. .

\section{Methods}

Individual sessions were offered in an outpatient setting, while both group and individual sessions occurred on an inpatient unit. This pilot study used a mixed methodology to collect data from young people who attended Bod Squad. A database of exercise physiology records for 47 young people were analysed for attendance and physiological indicators. In addition, seven semi-structured interviews were conducted with young people to explore their experience of Bod Squad.
\end{abstract}

\section{Results}

Young people attended a total of 169 sessions during the programs tenure, with an overall mean of 3.6 sessions. Pre-post measures for ten young people (who had attended at least four sessions) showed modest average reductions for body mass index, waist circumference, chest circumference and resting heart rate. Five themes emerged from the interviews -1) My reasons for attending, 2) The social aspect, 3) An individualised approach, 4) Outcomes from Bod Squad, and 5) My experience of the service. All of these themes included positive experiences of Bod Squad, which young people perceived as relevant to their needs and helpful to their recovery.

\section{Conclusion}

These findings are congruent with previous studies that have concluded that exercise physiology may be an effective, acceptable and valued intervention for addressing physical and metabolic health issues for young people.

\section{Key Words}

young adult, physical conditioning, physical fitness, adolescent psychiatry

This is the author manuscript accepted for publication and has undergone full peer review but has not been through the copyediting, typesetting, pagination and proofreading process, which may lead to differences between this version and the Version of Record. Please cite this article as doi: 10.1111/eip.12436

This article is protected by copyright. All rights reserved. 


\section{A Pilot Study of Client Outcomes from Exercise Physiology in a Youth Mental Health Service}

\section{Introduction}

It is well documented that people with mental illness are at higher risk of poorer physical health than those without mental illness (1-3). These risks may be serious enough to shorten life expectancy, with people experiencing mental illness dying between 10-30 years earlier than the general population (4). In an effort to address these risks, mental health services have become more focused on metabolic monitoring and physical health interventions (5).

Morgan et al. (6) report there is a growing interest in the effectiveness of exercise interventions for improving mental and physical health in individuals with mental illness, although supporting evidence has mostly arisen from studies with people with depressive disorders (7). Several studies have found exercise programs, along with other interventions that manage weight, enhance socialization and reduce cardiometabolic risk factors, assist with the management of these issues and enhance client well-being for people with mental illness $(2,3,8)$. A meta-analysis on lifestyle interventions has also confirmed that adjunctive non-pharmacological interventions were effective in reducing or attenuating antipsychoticinduced weight gain when compared to treatment-as-usual in patients with schizophrenia spectrum disorders, and that these effects were maintained at three months follow up (9).

Recently, a small but growing body of evidence around physical health and exercise for young people with first episode psychosis who are prescribed atypical antipsychotics has emerged. Curtis and colleagues $(5,10)$ report these young people commonly experience weight gain and obesity, with over a third either having metabolic syndrome or showing metabolic abnormalities. Symptoms related to first episode psychosis (e.g. decreased motivation, lack of volition, reduced participation in day-to-day activities ) and a higher prevalence of smoking also compound the risk of future cardiovascular disease. A pilot study has found that aerobic interval training effectively reduced waist circumference and resting heart rate, and increased VO2max for men with first episode psychosis (11), while a feasibility study has reported significant improvements in both physical and psychosocial outcomes from a 10 week exercise intervention with the same population (12). A recent phenomenological study explored the lived experience of young people aged 18-30 of participating in sports or exercise (13). Participants indicated that participation in exercise played an important role in their recovery from mental illness, often as a catalyst for practical change in their daily lives. They reported that exercise provided them with extra energy for activities of daily living (i.e. housework, community activities), and also gave them opportunities to engage in social interaction and communal interests. 
In response to these issues, Orygen Youth Health $(\mathrm{OYH})$ decided to explore opportunities for providing exercise programs for clients, with the clinical aim of improving both mental and physical outcomes. OYH is an early intervention mental health program in Melbourne, Australia, that provides specialist services for young people aged between 15-25 years of age. This includes crisis intervention and assessment, inpatient care, individual case management, intensive outreach services, family and consumer peer support and a comprehensive psychosocial group program. Up to 16 group programs are offered per week to assist young people to work on their goals, along with individual treatment. Improving physical health and appearance are commonly expressed goals for the young people who attend $\mathrm{OYH}$, indicating there is a client identified need for exercise and physical health programs. As expertise in exercise programs is limited amongst mental health clinicians (14), $\mathrm{OYH}$ sought to involve experts in this field for the development of a new program that focused on physical activity and health.

\section{Exercise Physiology in Mental Health}

Accredited Exercise Physiologists (AEP) specialise in the delivery of exercise for the prevention and management of chronic diseases and injuries (15). While the existence of a role for Exercise Physiologists in mental health was recognised at the turn of this century (16), the use of AEPs in mental health services remains in its infancy. Two studies have been published to date that explore the integration of exercise physiology into mental health services. A study of an exercise physiology program in an inpatient forensic setting in Western Australia (17) surveyed clients about their experiences of participating. The program combined exercises with education about the benefits of integrating physical activity into daily life. Clients reported that the program helped them to manage their psychiatric symptoms, and they also noted improvements in their fitness, confidence and self esteem.

A prospective, controlled study explored the introduction of a lifestyle and life skills intervention in two early psychosis community services (18). The intervention group received an individualised 3 month intervention, which included nurse, dietician and exercise physiologist input. In comparison to a control group receiving standard care, the intervention group achieved significantly less weight gain, and no significant increase in waist circumference. While the sample is relatively small $(n=28)$, the authors concluded this intervention was effective in attenuating weight gain for young people prescribed antipsychotic medication. Preliminary findings therefore indicate that exercise physiology may be an effective adjunctive means of addressing physical and metabolic health issues for young people, in a way that is acceptable to and valued by these clients. 


\section{Introducing 'Bod Squad'}

The introduction of exercise physiology into OYH was facilitated through a partnership with Australian Catholic University (ACU), who provides professional training in Exercise Physiology. A meeting between OYH and ACU was convened in 2014 to arrange the provision of an Accredited Exercise Physiologist employed by the university, to supervise an exercise physiology program with undergraduate exercise physiology students. The program was initially planned for two days per week over six months, with students on placement in pairs in six-week blocks. The program was subsequently extended for a further six-month period in 2015 , led by a different AEP but the same format of student placements. The AEP and students provided both group and individual sessions on an inpatient unit, and also provided individual sessions at the OYH community site, where a small gym was set up specifically for this program. Clients with a variety of diagnoses (including first -episode psychosis, borderline personality disorder and depression) were eligible for referral by their case managers, for assessment and to establish personal exercise plans. Prior to the program commencing, the OYH Youth Participation Group was consulted about strategies to engage young people. They suggested a youth friendly title for the program, which became known as "Bod Squad".

\section{Aim}

The aim of this pilot study was to evaluate the utilisation and experience of the Bod Squad exercise physiology program at $\mathrm{OYH}$. The research questions addressed were; 1) Who attended Bod Squad, and how often?, 2) What were the outcomes of participation for young people in regards to physical and physiological measurements? and 3) How did young people experience their attendance at Bod Squad?

\section{Method}

Ethical approval for this study was granted by the health service ethics committee (QA2014121). This pilot study used a mixed methodology that collected data from all stakeholders. Qualitative semi-structured interviews were conducted with both exercise physiology students and staff members from $\mathrm{OYH}$ and $\mathrm{ACU}$, and these findings are reported elsewhere. This article will report on data collected from young people participating in Bod Squad, from both quantitative data mining of clinical records and semi-structured interviews.

Bod Squad sessions commenced with assessments of variables such as fitness, strength and mobility, and a review for injuries. The exercise physiologist then collaboratively developed a program of exercises with the client, which was tailored to that individual's ability, goals and interests. All sessions were one hour in duration, and the program was adapted as required during the clients' attendance. Lifestyle changes and transferring the exercise program into 
everyday life were commonly addressed during discussion in the session, and exercise tasks were planned for completion between the sessions.

\section{Data Mining of Clinical Records}

Data mining uses statistical methods to identify patterns and trends in databases, and the use of that information for prediction and practice change (19). Data mining was used to address the first two research questions, using a database kept by both supervising AEPs which included data from 67 young people. A range of demographic variables were recorded in this database including gender, age and the number of sessions attended. In addition, the following physical and physiological measurements were recorded at baseline - body mass index (BMI), waist circumference (WC), hip circumference (HC), chest circumference (CC), and resting heart rate (RHR). For ten of the clients who attended The Bod Squad, these measurements were repeated after 4-8 sessions. Repeat measures were unavailable for other clients due to drop out, less than four sessions attendance or a decision by the client or exercise physiologist not to re-measure.

\section{Qualitative Semi-Structured Interviews}

Seven semi-structured interviews were conducted with young people to address the third research question. These interviews occurred at OYH immediately following exercise physiology sessions, after the young person had attended Bod Squad at least four times. The resulting transcript was analysed using content analysis (20), where themes were derived directly from the participants comments. The frequency of related comments were noted, grouped into similar responses and categorised under themes.

Several trustworthiness measures were employed to ensure the quality of the qualitative analysis. Each transcript was independently coded by two research team members, who then met to discuss the codes. A very high level of agreement was found, with $92 \%$ of codes either matching or being conceptually congruent. The remaining codes were discussed, and a consensus reached about their agreed meaning. A comprehensive audit trail was also kept, and the research team provided mutual peer review and supervision throughout.

\section{Results}

\section{Attendance at The Bod Squad}

The first session of Bod Squad occurred in July 2014, and the pilot program concluded in October 2015. However, appointments were only available from July - December 2014, and from May to October 2015. Clients attended a total of 164 sessions over that time, with a general trend found for greater attendance in the middle of the trial periods. Overall, 67 young people were referred to Bod Squad, with 47 going on to attend, a small majority of which $(n=38,57 \%)$ were male. The age of attendees ranged from 15 to 26 , with a mean of 
20.92 years. As illustrated in Figure 1, most of the clients attended between 1-3 sessions, with an overall mean of 3.6 sessions attended (Median $=3, \mathrm{SD}=2.96$ ).

\section{Place Figure 1 about here}

\section{Outcomes of Participation for Clients}

Of the ten clients for whom pre and post measurements were available, the genders were evenly split and their ages ranged from 16 to 25 . This group of clients attended an average of 6.4 sessions (range $4-8$ ), although the frequency of their attendance varied. Five of these participants attended weekly or fortnightly, while the other five attended irregularly. As shown in Table 1, modest average reductions across most variables were noted in this group, apart from measurements of hip circumference.

Place Table 1 about here

\section{Client Experiences of The Bod Squad}

Five themes emerged from the interviews with young people who had attended Bod Squad 1) My reasons for attending, 2) The social aspect, 3) An individualised approach, 4) Outcomes from Bod Squad, and 5) My experience of the service.

\section{My reasons for attending}

Clients identified a variety of reasons for attending Bod Squad, including developing greater strength, improving fitness and losing weight. These reasons were couched in terms of improving everyday function, and regaining some of the attributes they had lost in recent times; "It's nice to get back to being fit" (Interview 7). For one client, attendance at Bod Squad was specifically related to the physical consequences of their mental illness; "I've lost a lot of like muscle and that through an eating disorder, and I was looking to build it back, but didn't really have the motivation to do it by myself' (Interview 1). Several clients mentioned struggling with their motivation to attend Bod Squad consistently, although they found it easier to engage once they'd arrived at OYH. "Motivation was just like ..mmmm ... I don't really want to do this" (Interview 1). However, all of the clients interviewed had attended on at least four occasions, and had therefore been motivated enough by their reasons to attend to overcome these barriers.

\section{The Social Aspect}

Young people commented on the benefits of the social aspects of Bod Squad across both individual and group formats; "It just helps better my mood doing exercise with people that can help" (Interview 6). While some young people preferred 1:1 sessions, others who hadn't 
been on the inpatient unit expressed a desire to try group sessions, "It's a bit social as well ... I wasn't by myself you know ... you challenge the person next to you who can get the most ... they had two teams" (Interview 2)

\section{An individualised approach}

Regardless of the format, the young people really appreciated the individualised approach taken by the AEP and exercise physiology students towards their exercise program; "It's tailored to you" (Interview 1), "Everyone has personalised different programmes" (Interview 2). Young people indicated they found the alignment between their personal goals and the exercise programs they were given to be very satisfying and meaningful. The specialised knowledge of the AEP and exercise physiology students was also acknowledged by the students, who were impressed by both the breadth of their knowledge and how much they were willing to share; "Just sort of how knowledgeable they were about the whole, like about different [exercises] and everything. So if I had any sort of technical questions, they could convey it in a sort of layman's terms" (Interview 4). This knowledge gave the clients confidence about the programs value, and enabled them to increase their personal knowledge about physical health and exercise: "I feel good learning new things" (Interview 2).

\section{Outcomes from Bod Squad}

All of the young people perceived positive outcomes from Bod Squad, recognising beneficial impacts on both their physical and mental health; "There was a time when I was like so depressed, like I put it aside and didn't exercise at all. But I found it's a really good way to get like all my energy out, and it's something important [for] my life as well" (Interview 7). All of the clients reported reaching their personal goals, which promoted a deep sense of satisfaction and achievement; "The knowledge that I will be able to change myself for the better, no matter what happens ... I guess I could see a massive change from what my physical state was before to now" (Interview 6).

A major outcome for all of the young people was the incorporation of greater physical activity and exercise into their daily life. This is crucial to the clients continuing to benefit from this intervention into the future, and they all recognised the importance of keeping up with their new routines; "A take home exercise sheet, so l'm able to do that at home [and] a new set of exercises for when I transfer over to the gym" (Interview 4). This quote also illustrates how some young people also discussed linking with community based physical health services following the conclusion of Bod Squad.

\section{My Experience of The Service}

The young people had very positive experiences of working with the AEP and exercise physiology students, who they found engaging and helpful. The relative youth of the students was perceived to be an advantage, as they offered a peer based clinical experience; "I like 
having the students there instead of just like professional people, because it's kind of more fun with people close to your age and yeah, they're learning as well so it's not as strict, more comfortable" (Interview 1). Even though the students are novices, to the mental health field, young people still found the level of support they provided sufficient for their needs.

The only potential barriers to engaging in Bod Squad were related to the gym facilities at $\mathrm{OYH}$. Some of the equipment wasn't sufficient (e.g. computer speakers were too soft), while other equipment was recommended for purchase to expand the repertoire of exercises (e.g. a weights bench). The physical environment of the outpatient gym was also perceived as problematic; "Larger facility ... a bigger space with more open area for the exercises" (Interview 6), "The windows, oh my god it's so hot in here!" (Interview 7). Several clients also stated a desire for Bod Squad to be available every day year round, rather than the limited availability of this pilot.

\section{Discussion}

This study had addressed the proposed research question, and found that young people who attended Bod Squad experienced broadly positive outcomes. The Bod Squad was open to young people with a wide range of diagnoses (not just psychosis as in previous studies), and weight was not the only issue that motivated attendance at the program. The majority of young people attended between one and three sessions, although the reasons behind this limited engagement is unclear. Poor attendance and high dropout rates have been found in other studies of exercise interventions with adults with mental illness (21), with a lack of comfort and someone to go with identified as barriers. However, two prior studies with younger people reported relatively good engagement and adherence to exercise interventions $(12,18)$. The attendance of 67 young people at Bod Squad indicates that physical health and activity is a priority for these clients. However, young people in this study expressed a desire for group exercise, and the impact of this format on attendance should be explored in future studies.

The moderate average reductions in the variables were encouraging, indicating that even short engagement with exercise physiology could be beneficial. While similar positive outcomes were found by Curtis et al. (18), their study also incorporated nursing and dietetics intervention. Within mental health services, exercise physiology will be one of many disciplines who promote physical activity and exercise, but due to their expert knowledge they may be best positioned as leaders or consultants around this issue. There is a clear need for more rigorous and longitudinal studies into the impact of such programs on client health, and future studies must incorporate measures of mental health and wellbeing to fully capture impact and build on previous findings into the positive impact of exercise on mental health (12). 
Qualitative data from young people indicated their attendance at Bod Squad was a positive experience, which was directly relevant to their recovery. These findings consolidated those of Firth et al (22) who report an exercise intervention was positively experienced by people with first episode psychosis, producing benefits across a range of domains including improved confidence, a sense of achievement and decreased symptoms. It is important to elicit the perspectives and feedback of those who are both delivering and receiving exercise interventions for your people, to ensure that interventions are both acceptable and attractive (23), and more qualitative studies in this area are warranted. The presence of age contemporary health providers (i.e. exercise physiology students) appeared to contribute to the young peoples' perception of Bod Squad as a youth friendly service with friendly staff and less formality (24). This supports ongoing efforts to form partnerships with universities to provide opportunities for exercise physiology students to experience work in mental health, given the mutual benefit for both young people and the novice professionals.

As a pilot study, this study has several limitations which impact upon the transferability of its findings. The quantitative analysis was based on a relatively small sample size, recruited from the same service in a relatively limited geographical area. The participants who were interviewed had attended multiple sessions, and were therefore likely to be those who perceived the intervention positively. Interviewing participants who only attended once may have provided valuable information regarding attrition. However, the sample is adequate in relation to the qualitative analysis given this data focused on the lived experience of the young people. Participants also attended a limited number of exercise physiology sessions, due to a combination of attrition and the sporadic nature of this pilot program. Significant changes to health and wellbeing would not be expected from such limited exposure to this intervention, so the significance of the findings in the longer term cannot be determined without more rigorous investigation.

\section{Conclusion:}

This pilot study aimed to evaluate the utilisation and experience of Bod Squad, an exercise physiology program in a youth mental health service. While many people attended the program, their engagement was most often limited to three or less sessions and the positive outcomes reported here were only observed in a small sub-set of the sample. Despite this, modest reductions across several physiological risk indicators were recorded, and young people reported their experience of Bod Squad was both positive and rewarding. The findings presented here were congruent with previous studies that have concluded that exercise physiology may be an effective, acceptable and valued intervention for addressing physical and metabolic health issues for young people. While the limitations of this study have been 
acknowledged, the findings add to the existing preliminary basis for further investigations into the effectiveness of exercise physiology in youth mental health.

\section{References}

1. Correll C, Robinson D, Schooler N, Brunette M, Mueser K, Rosenheck R, et al. Cardiometabolic risk in patients with first-episode schizophrenia spectrum disorders. Baseline results from the RAISE-ETP study. JAMA Psychiatry 2014;71(12):1350-63.

2. Stanton R, Happell B. Exercise for mental illness: A systematic review of inpatient studies. International Journal of Mental Health Nursing. 2013;23:232-42.

3. Watkins A. Keeping the body in mind. ANMI Mental Health. 2014;21(11):44-5.

4. Holt RIG, Mitchell AJ. Diabetes mellitus and severe mental illness: mechanisms and clinical implications. Nat Rev Endocrinol. 2015;11(2):79-89.

5. Curtis J, Henry C, Watkins A, Newall H, Samaras K, P W. Metabolic abnormalities in an early psychosis service: a retrospective, naturalistic cross-sectional study. Early Intervention in Psychiatry. 2011;5:108-14.

6. Morgan A, Parker A, Alvarez-Jimenez M, Jorm A. Exercise and Mental Health: An Exercise and Sports Science Australia Commissioned Review. Journal of Exercise Physiology Online. 2013;16(4):64-73.

7. Rosenbaum S, Tiedemann A, Sherrington C, Curtis J, Ward P. Physical activity interventions for people with mental illness: a systematic review and meta-analysis. Journal of Clinical Psychiatry. 2014;75(9):964-74.

8. Stanton R. Accredited exercise physiologists and the treatment of people with mental illnesses. Clinical Practice. 2013;2(2):5-9.

9. Alvarez-Jimenez M, Hetrick S, Gonzalez-Blanch C, Gleeson J, McGorry P. Nonpharmacological management of antipsychotic-induced weight gain: systematic review and meta-analysis of randomised controlled trials. British Journal of Psychiatry. 2008;193(2):1017.

10. Curtis J, Newall H, Samaras K. The heart of the matter: cardiometabolic care in youth with psychosis. Early Intervention in Psychiatry. 2012;6(3):347-53.

11. Abdel-Baki, A, BrazziniPoisson, V, Marois, F, Letendre, E, Karelis, AD. Effects of aerobic interval training on metabolic complications and cardiorespiratory fitness in young adults with psychotic disorders: a pilot study. Schizophrenia research. 2013;149(1):112-5. 12. Firth, J, Carney, R, Elliott, R, French, P, Parker, S, Mclntyre, R, McPhee, JS, Yung, AR. Exercise as an intervention for first-episode psychosis: a feasibility study, Early Intervention in Psychiatry. 2016;doi: 10.1111/eip.12329 .

13. Staal A, Jespersen $E$. The lived experiences of participating in physical activity among young people with mental health problems: A recovery-oriented perspective. Physical Culture and Sport Studies and Research. 2015;65:41-50.

14. Stanton RBP. Investigating the exercise-prescription practices of nurses working in inpatient mental health settings. International Journal of Mental Health Nursing.

2015;24(2):112-20.

15. Exercise and Sports Science Australia. How can an Accredited Exercise Physiologist help? 2014. Available from: http://www.essa.org.au/essa-me/how-can-an-accreditedexercise-physiologist-help/.

16. Wattles MG. The Role of the Exercise Physiologist in Mental Health. Professionalization of Exercise Physiology. 2001;4(4):2-.

17. Wynaden D, Barr L, Omari O, Fulton A. Evaluation of service users' experiences of participating in an exercise programme at the Western Australian State Forensic Mental Health Services. International Journal of Mental Health Nursing. 2012;21(3):229-35 7p.

18. Curtis J, Watkins A, Rosenbaum S, Teasdale S, Kalucy M, Samaras K, et al. Evaluating an individualized lifestyle and life skills intervention to prevent antipsychoticinduced weight gain in first-episode psychosis. Early Intervention in Psychiatry. 2015:n/a-n/a. 
19. Kincade K. Data mining: digging for healthcare gold. Insurance \& Technology. 1998;23(2):IM2-IM7.

20. Vaismoradi $\mathrm{M}$, Turunen $\mathrm{H}$, Bondas $\mathrm{T}$. Content analysis and thematic analysis: Implications for conducting a qualitative descriptive study. Nursing \& Health Sciences. 2013;15:398-405.

21. Archie S, Wilson J, Osborne S, Hobbs H, McNiven J. Pilot Study: Access to Fitness Facility and Exercise Levels in Olanzapine-Treated Patients. Canadian Journal of Psychatty. 2003;48:628-32.

22. van Sluijs E, Kriemler S. Reflections on physical activity intervention research in young people - dos, don'ts, and critical thoughts

. International Journal of Behavioral Nutrition and Physical Activity. 2016;13:25.

23. Ambresin A, Bennett K, Patton G, Sanci L, Sawyer S. Assessment of Youth-Friendly Health Care: A Systematic Review of Indicators Drawn From Young People's Perspectives. Journal of Adolescent Health. 2013;52(6):670-81.

\section{Tables}

\begin{tabular}{|l|l|l|l|}
\hline Measurement & First session (Mean) & $\begin{array}{l}\text { Final session } \\
\text { (Mean) }\end{array}$ & Difference \\
\hline $\mathrm{BMI}$ & 27.92 & 27.4 & -0.52 \\
\hline $\mathrm{WC}$ & $95.00 \mathrm{~cm}$ & $92.37 \mathrm{~cm}$ & $-2.63 \mathrm{~cm}$ \\
\hline $\mathrm{HC}$ & $106.62 \mathrm{~cm}$ & $107.62 \mathrm{~cm}$ & $+1.00 \mathrm{~cm}$ \\
\hline $\mathrm{CC}$ & $115 \mathrm{~cm}$ & $111.5 \mathrm{~cm}$ & $-3.5 \mathrm{~cm}$ \\
\hline $\mathrm{RHR}$ & $82 \mathrm{bpm}$ & $77 \mathrm{bpm}$ & $-5 \mathrm{bpm}$ \\
\hline
\end{tabular}

Table 1: Physical Outcomes for Clients

\section{Figure Legends}

Figure 1: Total Number of Sessions Attended Per Patient

This article is protected by copyright. All rights reserved. 


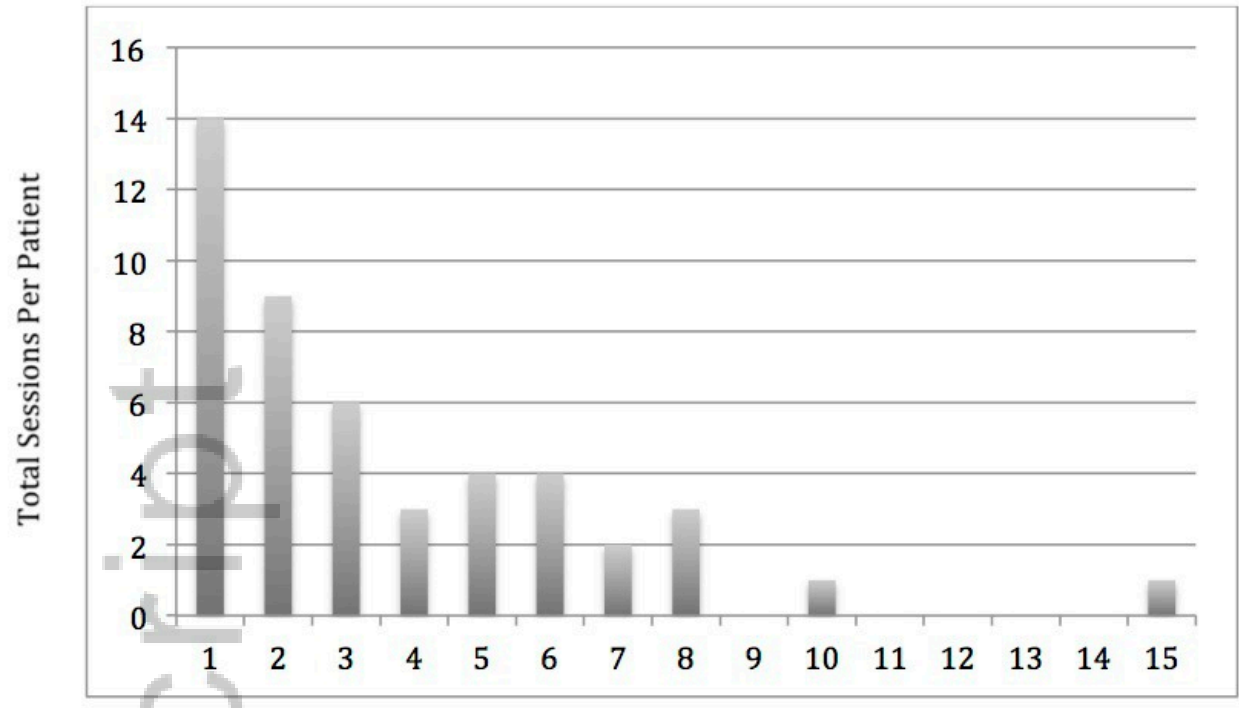

Number of Sessions Attended

Figure 1: Total Number of Sessions Attended Per Patient

Figure 1.jpg

This article is protected by copyright. All rights reserved. 
Title:

A Pilot Study of Client Outcomes from Exercise Physiology in a Youth Mental Health Service

Authors:

Gina Woodhead, Danielle Hitch, Kate Bolton, Dianne Albiston \& Eoin Killackey

\section{Correspondence:}

Dr. Danielle Hitch

Psychosocial Research Centre, 130 Bell Street, Coburg VIC 3058

Email: Danielle.Hitch@mh.org.au

Phone: 0393559832

Acknowledgements: The research team would like to acknowledge the assistance and support of the Exercise Physiology program at Australian Catholic University, without whom this study would not have been possible

This article is protected by copyright. All rights reserved. 


\section{University Library}

\section{- M M I N E R VA A gateway to Melbourne's research publications}

Minerva Access is the Institutional Repository of The University of Melbourne

Author/s:

Woodhead, G;Hitch, D;Bolton, K;Albiston, D;Killackey, E

Title:

Pilot study of client outcomes from exercise physiology in a youth mental health service

Date:

2018-08-01

Citation:

Woodhead, G., Hitch, D., Bolton, K., Albiston, D. \& Killackey, E. (2018). Pilot study of client outcomes from exercise physiology in a youth mental health service. EARLY INTERVENTION IN PSYCHIATRY, 12 (4), pp.734-739. https://doi.org/10.1111/eip.12436.

Persistent Link:

http://hdl.handle.net/11343/292910 